\begin{tabular}{|c|l|}
\hline Title & $\begin{array}{l}\text { In Situ Optical Second Harmonic Rotational A nisotropy Measurements of an Au(111) Electrode during Electrochemical } \\
\text { Deposition of Tellurium }\end{array}$ \\
\hline Author(s) & Y agi, Ichizo; Nakabay ashi, Seiichiro; Uosaki, Kohei \\
\hline Citation & $\begin{array}{l}\text { Journal of Physical Chemistry B, 102(15), 2677-2683 } \\
\text { https:/doi.org/10.1021/jp972763n }\end{array}$ \\
\hline Issue Date & 1998_0409 \\
\hline Doc URL & http://hdl.handle.net/2115/50242 \\
\hline Type & article \\
\hline File Information & JPCB102-15_2677-2683.pdf \\
\hline
\end{tabular}

Instructions for use 


\title{
In Situ Optical Second Harmonic Rotational Anisotropy Measurements of an Au(111) Electrode during Electrochemical Deposition of Tellurium
}

\author{
Ichizo Yagi, ${ }^{\dagger}$ Seiichiro Nakabayashi, ${ }^{\ddagger}$ and Kohei Uosaki* \\ Physical Chemistry Laboratory, Division of Chemistry, Graduate School of Science, Hokkaido University, \\ Sapporo 060, Japan
}

Received: August 25, 1997; In Final Form: December 18, 1997

\begin{abstract}
The electrodeposition of Te on a single-crystalline $\mathrm{Au}(111)$ electrode was studied with 1064-nm-excited SH rotational anisotropy measurements. The SH rotational anisotropy was significantly changed with the first underpotential deposition (upd) of Te, and the bulk Te deposition attenuated the anisotropic character of the overall surface symmetry. The change in the $\mathrm{SH}$ rotational anisotropy during the first upd of Te was examined using two different models. The first model considered only the contribution of the $\mathrm{Au}(111)$ surface to the SHG response, while the second one took into account the contributions of both the Au(111) substrate and the adsorbed Te layer. In the former case, the change in the SH rotational anisotropy can be explained by considering the quenching of the nonlinear susceptibility of the $\mathrm{Au}(111)$ surface. The analysis based on the latter model resulted in a rotation angle of 608 for the adsorbed Te layer against the the $\mathrm{Au}(111)$ lattice. This value was not consistent with that expected from the adsorbate structure of the first upd layer of Te, i.e., $(\sqrt{ } 3 \times \sqrt{ } 3) R 30^{\circ}$. Thus, the former model seems to be more appropriate to explain the present results. The SH rotational anisotropy measurement also suggests that the morphology became more isotropic after the bulk Te deposition.
\end{abstract}

\section{Introduction}

Adsorption and deposition processes at surfaces are the initial steps of crystal growth and govern the resultant structure of the films. For the growth of the films with well-defined structures, a uniform and defect-free structure of the initial deposit is required. Underpotential deposition (upd), which is the electrochemical deposition of a metal onto a foreign substrate at potentials more positive than the corresponding Nernst potential for bulk deposition (e.g., for $\mathrm{Cu}$ on $\mathrm{Cu}$ ), generally results in the formation of a well-defined adlayer of the deposited metal. Upd has been studied in great detail and has been observed for many pairs of upd metals and substrates. ${ }^{1-3}$ Recently, upd has been used as a common method to prepare structurally welldefined atomic layers in the electrochemical environment. ${ }^{4-10}$ The electrochemical atomic layer epitaxy (ECALE), which is an analogue of ALE in the gas phase of binary materials, is achieved by sequential upd of two different elements. Stickney and his co-workers used this technique to prepare CdTe, CdS, $\mathrm{CdSe}$, and GaAs films on gold electrode surfaces. ${ }^{11-20}$

In the previous studies of the ECALE of CdTe on the lowindex plane of $\mathrm{Au},{ }^{13-16}$ however, a large number of Te islands, which were deposited as the first deposit, were observed. The island structure of the Te upd layer should be avoided in order to grow epitaxial layers of $\mathrm{Cd}$ and $\mathrm{Te}$. To control the structure of the Te upd layer, it is essential to understand both the initial growth mechanism of the upd layer and the reactivity of the Te-covered Au surface. Although scanning probe microscopes (SPMs) are very powerful tools to examine the structure of the

* Corresponding author. Fax: +81-11-706-3440. E-mail: uosaki@ pcl.chem.hokudai.ac.jp

$\dagger$ Present address: Department of Applied Chemistry, Faculty of Engineering, The University of Tokyo, Tokyo 113, Japan.

$\stackrel{+}{\lessgtr}$ Present address: Department of Chemistry, Faculty of Science, Saitama University, Urawa 338, Japan. complete upd layer, ${ }^{15,21,22}$ in situ monitoring of the initial deposition step by these techniques is rather difficult because these techniques have relatively low time resolution and usually require a very highly ordered structure to obtain atomically resolved images. Ex situ techniques in UHV such as LEED ${ }^{13,14}$ are also not suitable for the dynamic study, since they require the transfer of a sample electrode from the electrochemical cell into the UHV chamber.

Optical second harmonic generation (SHG) has proven to be a useful technique to study adsorption processes and growth mechanisms. ${ }^{23-26}$ For the study of electrochemical interfaces, this method has received wide attention because of its unique sensitivity to interfacial properties. By using the SHG technique, information about the surface electronic and geometric structures can be obtained in real time and in the electrochemical environment. In our previous study, we demonstrated that the adsorption of Te has a great impact on the electronic structure of a gold surface, and therefore, the electrochemical deposition of Te on an Au electrode can be monitored by observing the variation in the SHG response. ${ }^{27}$

While SHG measurements at isotropic surfaces such as a polycrystalline surface, however, do not provide geometrical information, SH rotational anisotropy measurements on singlecrystal surfaces, where the orientation of atoms is defined, can provide information about the symmetry at the surface at the atomic level. Thus, more details about the processes of electrochemical deposition can be clarified from the SHG measurements using a single-crystalline substrate.

In the present study, the electrochemical deposition of Te on a $\mathrm{Au}(111)$ single-crystal electrode was studied at the single excitation wavelength of $1064 \mathrm{~nm}$. The SH rotational anisotropy was significantly changed during the deposition of Te. Two different models were considered in order to explain the change 
in the SH rotational patterns and strong influence of Te atoms on the surface electronic structure of the $\mathrm{Au}(111)$ surface.

\section{Theory}

According to the formalism of Sipe et al., the total anisotropy for the SH intensity, $I(2 \omega)$, upon the azimuthal rotation of the $C_{3 v}$ surface is given by ${ }^{28,29}$

$$
\begin{gathered}
I_{\mathrm{pp}}(2 \omega)=\left|\mathbf{a}_{\mathrm{pp}}^{(\infty)}+\mathbf{d}_{\mathrm{pp}}^{(3)} \cos (3 \phi)\right|^{2} I_{\mathrm{p}}(\omega)^{2} \\
I_{\mathrm{sp}}(2 \omega)=\left|\mathbf{a}_{\mathrm{sp}}^{(\infty)}+\mathbf{d}_{\mathrm{sp}}^{(3)} \cos (3 \phi)\right|^{2} I_{\mathrm{s}}(\omega)^{2} \\
I_{\mathrm{ps}}(2 \omega)=\left|\mathbf{d}_{\mathrm{ps}}^{(3)} \sin (3 \phi)\right|^{2} I_{\mathrm{p}}(\omega)^{2} \\
I_{\mathrm{ss}}(2 \omega)=\left|\mathbf{d}_{\mathrm{ss}}^{(3)} \sin (3 \phi)\right|^{2} I_{\mathrm{s}}(\omega)^{2}
\end{gathered}
$$

where $\phi$ is the azimuthal angle that is defined as the angle between a certain crystal axis of the sample surface and the direction of beam propagation parallel to the surface, and $\mathbf{a}^{(\infty)}$ and $\mathbf{d}^{(3)}$ represent the rotational constants composed of linear combinations of surface and bulk tensor elements. The superscripts of the constants indicate the associated azimuthal dependence. Thus, $\mathbf{a}^{(\infty)}$ and $\mathbf{d}^{(3)}$ are associated with the isotropic and 3-fold contributions, respectively. The subscripts on the constants indicate their association to the input and output polarizations given by the first and second indices, respectively. The Fresnel factors are included in these constants. ${ }^{29}$ These functions imply that the p-polarized SH intensities, $I_{\mathrm{pp}}(2 \omega)$ and $I_{\mathrm{sp}}(2 \omega)$, will have 3 -fold dependence on the azimuthal rotation of the surface. The exact form depends on the magnitudes and phases of the tensor elements that are included in the constants. The s-polarized SH intensities, $I_{\mathrm{ps}}(2 \omega)$ and $I_{\mathrm{ss}}(2 \omega)$, will always result in a perfect 6-fold dependence upon the azimuthal rotation. In all cases, the azimuthal angles where the maxima and minima of the SH intensity occur must correspond to those where the direction of the $k$ vector agrees with either the $x$ or $y$ surface crystal axis direction.

Distortion of the $\mathrm{SH}$ rotational patterns of the $C_{3 v}$ surface from the perfect 3-fold or 6-fold symmetry indicates a change in the symmetry class of the surface or the addition of a different phase with different symmetry. For example, the reconstruction of $\mathrm{Au}(111)$ causes a one-dimensional distortion of the $\mathrm{SH}$ rotational patterns. This change was attributed to a change in the surface symmetry from $C_{3 v}$ to $C_{s}$.

Although the $\mathrm{SH}$ rotational anisotropy pattern of the $C_{3 v}$ surface for the $\mathrm{p}$-in/p-out optical polarization combination is expected to have 3-fold symmetry as already mentioned, it results in various patterns depending on the ratio between $\mathbf{a}_{\mathrm{pp}}{ }^{(\infty)}$ and $\mathbf{d}_{\mathrm{pp}}{ }^{(3)}$. This is caused by the interference between the isotropic $\mathrm{SH}$ polarization term, $\mathbf{a}_{\mathrm{pp}}{ }^{(\infty)}$, and the anisotropic $\mathrm{SH}$ polarization term, $\mathbf{d}_{\mathrm{pp}}{ }^{(3)}$, in eq 1a. The terms $\mathbf{a}_{\mathrm{pp}}{ }^{(\infty)}$ and $\mathbf{d}_{\mathrm{pp}}{ }^{(3)}$ contain surface second-order nonlinear susceptibility elements, $\chi^{(2)}{ }_{i j k}$, higher order bulk susceptibility terms, $\gamma$ and $\xi$, and the Fresnel factors. While the coefficient $\mathbf{a}_{\mathrm{pp}}{ }^{(\infty)}$ contains the isotropic SH polarization due to $\chi^{(2)}{ }_{z z z}, \chi^{(2)}{ }_{z i i}$, and $\chi^{(2)}{ }_{i i z}, i=(\mathrm{x}, y)$, and $\gamma$, the coefficient $\mathbf{d}_{\mathrm{pp}}{ }^{(3)}$ contains the in-plane $\mathrm{SH}$ polarization due to $\chi^{(2)}{ }_{x x x}$ and $\xi$. The former susceptibility elements are nonzero even at the isotropic surfaces and, then, are referred to as isotropic susceptibility elements. The isotropic SH polarization term, $\mathbf{a}_{\mathrm{pp}}{ }^{(\infty)}$, has no dependence on the azimuthal angle of rotation. Thus, the observed rotational anisotropy arises from the latter susceptibility elements, and they are then referred to as anisotropic susceptibility elements. The anisotropic SH polarization term, $\mathbf{d}_{\mathrm{pp}}{ }^{(3)}$, for $C_{3 v}$ symmetry possesses, therefore, three maxima and minima during a rotation. The $\mathrm{SH}$ rotational patterns can be understood by considering how these two terms interfere with each other. ${ }^{8}$ A fit of the $\mathrm{SH}$ rotational data to eq 1a yields the ratio $\mathbf{d}_{\mathrm{pp}}{ }^{(3)} / \mathbf{a}_{\mathrm{pp}}{ }^{(\infty)}$, which contains a magnitude and relative phase angle reflecting this interference under the given experimental conditions. Rotational anisotropy measurements under the s-in/p-out optical polarization combination are also sensitive to the relative phase shift.

The physical meaning of the phase shift was briefly mentioned by Pettinger et al. and may be evident by considering a two-level system. ${ }^{31,32}$ In a nonresonant situation where the $2 \omega$ energy does not match the energy difference between the two levels, the SHG process can be considered as a two-photon absorption/one-photon emission process via a pseudostate, and the virtually transitted electron can occupy an upper state for a very short interaction time, $\Delta t$, which is determined by the energy mismatch $\Delta E$. In the frame of the Heisenberg uncertainty law, the allowed interaction time is given by $\Delta t \leq \hbar / \Delta E$. Thus, in the nonresonant condition where $\Delta E$ is large, $\Delta t$ should be short and then the phase shift, $\delta$, is small. The more resonant the transition, the larger the phase shift. The value of $\delta$ is the absolute phase shift, which is defined as the difference between the phase of the fundamental light and that of the SH light. Since both the isotropic and anisotropic terms in eqs $1 \mathrm{a}$ and $1 \mathrm{~b}$ contain many nonlinear susceptibility tensor elements, if resonance occurs for a given tensor element, the value of $\delta$ should change, and as a result, the relative phase difference between the terms $\mathbf{a}^{(\infty)}$ and $\mathbf{d}^{(3)}$ changes. The quantitative evaluation, however, is difficult since the Fresnel factors describing the effective electric field at metal surfaces are complex; they also bring changes of the phases of both $\mathbf{a}_{\mathrm{pp}}{ }^{(\infty)}$ and $\mathbf{d}_{\mathrm{pp}}{ }^{(3)}$. 31

\section{Experimental Section}

Chemicals. Water purified by a Milli-Q purification system (Millipore) was used for the preparation of the electrolyte solutions and solutions for the surface preparation in all experiments. Perchloric acid $\left(\mathrm{HClO}_{4}, 60 \%\right)$ was reagent grade and was used as received from Wako Pure Chemicals. The solution containing tellurium was prepared by dissolving $\mathrm{TeO}_{2}$ (99.995\%, Aldrich Chemicals) in concentrated perchloric acid and diluted to a suitable concentration for the experiments. The electrolyte solutions were deaerated by purging with high-purity nitrogen ( $>99.99 \%$ ) before each experiment.

Preparation of the Electrode. A commercially available $\mathrm{Au}(111)$ single-crystal disk (10 mm diameter, $3 \mathrm{~mm}$ thickness, MaTeck) was used as an electrode. The surface was polished with alumina powder, using successively smaller grades down to $0.05 \mu \mathrm{m}$, to be optically flat and was sonicated in Milli-Q water. The electrode was dipped in concentrated sulfuric acid for $12 \mathrm{~h}$ and it was then annealed in an electric furnace at 800 ${ }^{\circ} \mathrm{C}$ for 5 hours. A final treatment for electrochemical SHG measurements was performed following the method of Clavilier et al. $^{33}$ and Motoo and Furuya. ${ }^{34}$ The single crystal was annealed in a gas-oxygen flame near $800{ }^{\circ} \mathrm{C}$ for $30 \mathrm{~s}$ and was quickly brought into nitrogen-saturated ultrapure water. The surface was covered with a droplet of water while the crystal was mounted in a Kel-F holder and transferred to a spectroelectrochemical cell.

Spectroelectrochemical Cell. The single-window spectroelectrochemical cell was made from a cylindrical Kel-F block. The sample electrode holder assembly was inserted into the electrochemical compartment of the spectroelectrochemical cell so that rotation of the electrode surface was possible. An O-ring 
was held around the front part of the holder assembly to provide the solution seal and the rotation gasket.

Experimental Arrangement for Electrochemical SHG Measurement. The $10 \mathrm{~ns}$ pulses of fundamental light (1064 $\mathrm{nm}$ ) generated by a $10 \mathrm{~Hz}$ Q-switched Nd:YAG laser (LEXEL HY-200S) were directed onto the surface of the gold electrode at a $35^{\circ}$ angle of incidence in the single-window spectroelectrochemical cell, which was mounted on an $x, y, z, \theta$ translation stage to allow the placement of the crystal. The optical arrangement and the detection system for SHG were essentially the same as that for the near-infrared excitation measurement given in the previous report. ${ }^{27}$

For the electrochemical measurement, a standard threeelectrode configuration was constructed with a $\mathrm{Ag} / \mathrm{AgCl}$ (saturated $\mathrm{NaCl}$ ) reference electrode and a $\mathrm{Pt}$ counter electrode. The electrode potential was controlled with an electric polarization unit (Toho Tech. Res., PS-07). The electrode potential, the current, and the normalized SH intensity were digitally captured via a computer interface module (Stanford Research, SR245) and a GP-IB board (NEC, PC-9801-29n) and stored on a personal computer (NEC, PC-9801RS).

Electrochemical SHG measurements were carried out as follows. First, cyclic voltammetry (CV) was performed on a bare gold electrode by sweeping the potential between $-0.2 \mathrm{~V}$ and $+1.5 \mathrm{~V}$ in $0.1 \mathrm{M} \mathrm{HClO}_{4}$ solution $(1$ cycle, sweep rate $=$ $50 \mathrm{mV} \mathrm{s}^{-1}$ ) to confirm that the $\mathrm{CV}$ that was typical of a clean $\mathrm{Au}(111)$ electrode was obtained. After a clean gold surface was obtained, the deaerated electrolyte solution containing tellurium was introduced into the spectroelectrochemical cell with nitrogen pressure. To prevent the adsorption of Te on the Au surface, the potential was kept at $+0.8 \mathrm{~V}$ during exchange of the solution in the spectroelectrochemical cell. The electrode potential was then swept and the SHG measurements were started.

The SH rotational anisotropy was obtained by measuring the SH intensity from the electrode surface as a function of the rotation angle of the surface about the surface normal. The azimuthal angle was defined as the angle between the plane of incidence for the fundamental and the $[2-1-1]$ axis of the $\mathrm{Au}(111)$ surface. The electrode potential was set at a constant value during the rotational anisotropy measurements. The $\mathrm{SH}$ intensity was integrated over 30 laser shots for each $5^{\circ}$ angle of rotation. The data acquisition was controlled by a personal computer. The obtained SH rotational anisotropy patterns were fitted to the theoretical equations such as eqs $1 \mathrm{a}-1 \mathrm{~d}$ which were derived for various polarization conditions and surface symmetries, $C_{3 v}$.

\section{Results}

4.1. Electrochemistry of Te Deposition. Figure 1a shows a $\mathrm{CV}$ of the $\mathrm{Au}(111)$ electrode in $0.1 \mathrm{M} \mathrm{HClO}_{4}$ solution. The shape of the curve was in good agreement with the CVs of Au(111) electrodes reported in the literature. Figure $1 \mathrm{~b}$ shows a $\mathrm{CV}$ of the $\mathrm{Au}(111)$ electrode in $0.1 \mathrm{M} \mathrm{HClO}_{4}$ solution containing $0.5 \mathrm{mM} \mathrm{TeO}$. During the negative potential scan from the initial potential, $+800 \mathrm{mV}$, a cathodic current corresponding to the first upd of Te started to flow around $+400 \mathrm{mV}$. The second upd peak, clearly resolved from the bulk Te deposition current, appeared at $+50 \mathrm{mV}$. In the positive scan, the stripping peaks corresponding to the dissolution of the bulk, second upd and first upd layers of Te were observed at $+400,470$, and $560 \mathrm{mV}$, respectively. As a comparison, a CV of a polycrystalline $\mathrm{Au}$ electrode in $0.1 \mathrm{M} \mathrm{HClO}_{4}$ solution containing 0.5 $\mathrm{mM} \mathrm{TeO} 2$ is also shown in Figure 1c. Although the electro-

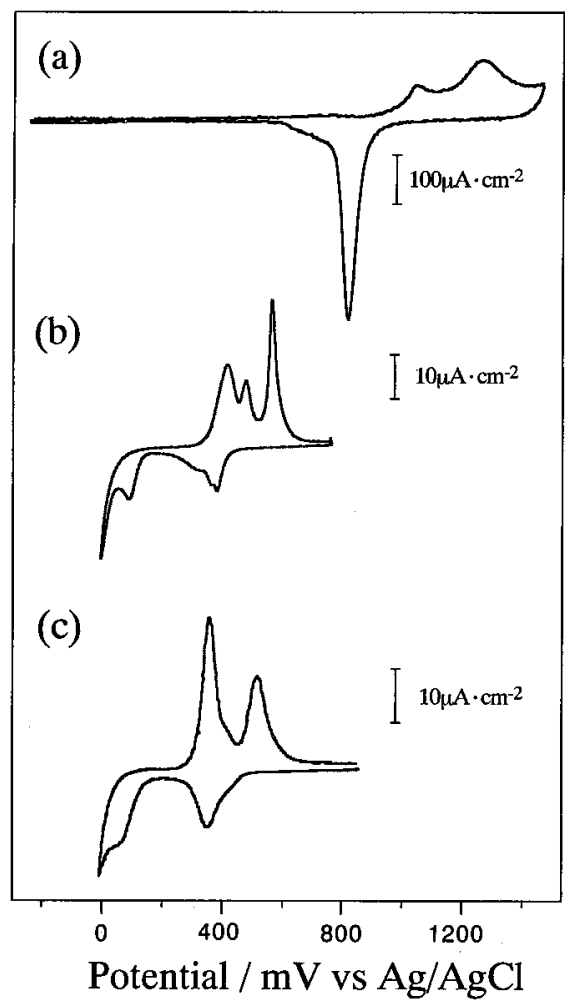

Figure 1. Cyclic voltammograms of $\mathrm{Au}$ electrodes: (a) $\mathrm{Au}(111)$ electrode in $0.1 \mathrm{M} \mathrm{HClO}_{4}$, scan rate $50 \mathrm{mV} \mathrm{s}^{-1}$; (b) $\mathrm{Au}(111)$ electrode in $0.1 \mathrm{M} \mathrm{HClO}_{4}+0.5 \mathrm{mM} \mathrm{TeO}_{2}$, scan rate $5 \mathrm{mV} \mathrm{s}^{-1}$; (c) Au(poly) electrode in $0.1 \mathrm{M} \mathrm{HClO}_{4}+0.5 \mathrm{mM} \mathrm{TeO}_{2}$, scan rate $5 \mathrm{mV} \mathrm{s}^{-1}$.

TABLE 1: Summary of the Coverages of Te Atoms at Various Deposition Steps ${ }^{a}$

\begin{tabular}{clccc}
\hline face & peak & $\begin{array}{c}\text { coverage } \\
\left(\times 10^{15} \mathrm{~cm}^{-2}\right)\end{array}$ & $\begin{array}{c}\text { ideal coverage } \\
\left(\times 10^{15} \mathrm{~cm}^{-2}\right)\end{array}$ & $\begin{array}{c}\text { LEED } \\
\text { pattern }\end{array}$ \\
\hline $\begin{array}{c}\text { previous work } \\
\mathrm{Au}(111)\end{array}$ & 1st upd & 0.48 & 0.45 & $(12 \times 12)$ \\
& 2nd upd & 0.64 & 0.60 & $(3 \times 3)$ \\
bulk & 3.45 & & \\
present work & & & & \\
$\mathrm{Au}(111)$ & 1st upd & 0.47 & & \\
& 2nd upd & 0.71 & & \\
$\mathrm{Au}($ poly) & 1st upd & 0.47 & & \\
& 2nd upd & 0.88 & &
\end{tabular}

${ }^{a}$ Coverages are given in Te atoms $/ \mathrm{cm}^{2}$. The coverages in the previous work (ref 15) were determined by basing on the observed AES current for Te where the conversion factor of $5.03 \times 10^{13}$ was used. In the present work, coverages are determined from the Faradic charge density during each desorption of Te.

chemical behavior of $\mathrm{Au}(111)$ was similar to that of a polycrystalline Au electrode, the peaks of $\mathrm{Au}(111)$ are sharper than those of $\mathrm{Au}$ (poly). Similar CVs were reported in previous studies using $\mathrm{Au}$ single-crystal surfaces ${ }^{6,21}$ and polycrystalline Au. ${ }^{7-10,26}$ The reductive deposition of $\mathrm{Te}$ from $\mathrm{TeO}_{2}$ is known to occur by the following four-electron reduction process: ${ }^{11-16,35-37}$

$$
\mathrm{HTeO}_{2}^{+}+3 \mathrm{H}^{+}+4 \mathrm{e}^{-} \rightarrow \mathrm{Te}+2 \mathrm{H}_{2} \mathrm{O}
$$

Table 1 summarizes the electric charge that flowed during each step of the Te desorption and the surface coverage of Te calculated from the charge at both the $\mathrm{Au}(111)$ and $\mathrm{Au}($ poly) electrodes. These values are similar to those reported by Stickney and his co-workers, ${ }^{15}$ which are also shown in this table. 

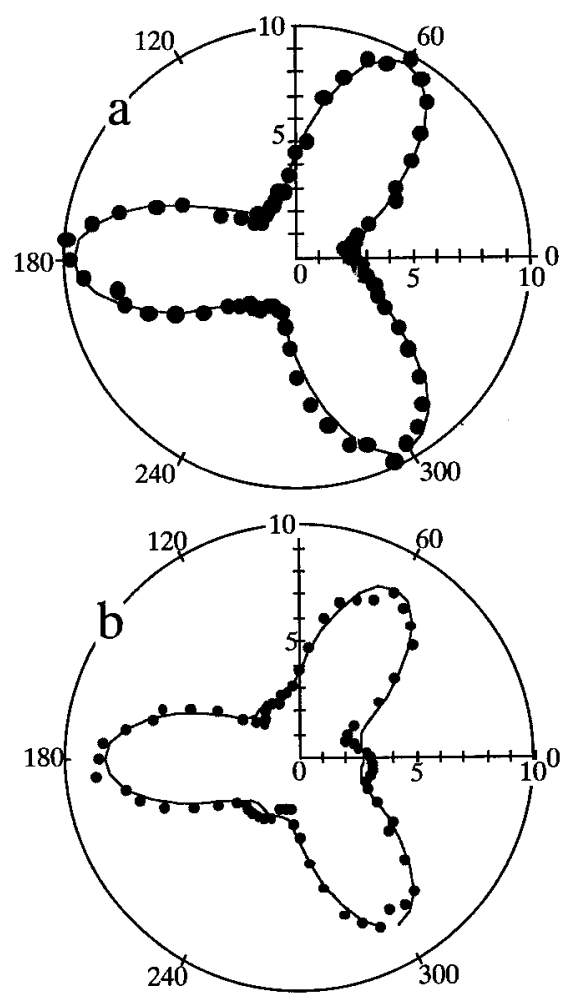

Figure 2. SH rotational patterns of the $\mathrm{Au}(111)$ electrode surface in $0.1 \mathrm{M} \mathrm{HClO}_{4}$ at (a) $+800 \mathrm{mV}$ and (b) $+150 \mathrm{mV}$. Polarization: $\mathrm{p}$-in/ p-out. Circles show data points, and solid line indicates the theoretical fit.

4.2. SH Rotational Anisotropy. Figure 2 shows the p-in/ p-out $\mathrm{SH}$ rotational anisotropy patterns of the $\mathrm{Au}(111)$ electrode biased at (a) $+800 \mathrm{mV}$ and (b) $+150 \mathrm{mV}$ in $0.1 \mathrm{M} \mathrm{HClO}_{4}$. Although both patterns showed the 3-fold symmetry with the $\mathrm{SH}$ intensity maxima at the azimuthal angles of $60^{\circ}, 180^{\circ}$, and $300^{\circ}$, the $\mathrm{SH}$ intensities at $+150 \mathrm{mV}$ were smaller than those at $+800 \mathrm{mV}$. The $\mathrm{SH}$ rotational anisotropy pattern gradually shrank as the potential became more negative. The onedimensional distortion of the $\mathrm{SH}$ pattern, which was reported for the reconstructed $\mathrm{Au}(111)$ in the negative potential region by Pettinger et al., ${ }^{38-40}$ was not observed in the present study.

The p-in/p-out SH rotational patterns shown in Figure 3 were obtained in $0.1 \mathrm{M} \mathrm{HClO}_{4}$ solution containing $0.5 \mathrm{mM} \mathrm{TeO}_{2}$ at various potentials where each deposition process of Te was completed (cf. Figure $1 \mathrm{~b}$ ). Figure $3 \mathrm{a}$ is the $\mathrm{SH}$ rotational pattern obtained at $+800 \mathrm{mV}$, where Te deposition does not occur. Nearly the same 3 -fold $\mathrm{SH}$ rotational pattern as that in Figure $3 \mathrm{a}$ was obtained, clearly indicating that the bare Au(111) surface was exposed at this potential. Figure $3 b$ shows the $\mathrm{SH}$ rotational pattern at $+150 \mathrm{mV}$, where the first upd of Te finished and the ordered structure of adsorbed Te was expected. ${ }^{15}$ The original 3 -fold pattern shown in Figure 3a significantly shrank and another 3-fold rotational pattern appeared with the maxima at azimuthal angles of $0^{\circ}, 120^{\circ}$, and $240^{\circ}$. Figure $3 \mathrm{c}$ is the $\mathrm{SH}$ rotational pattern obtained at +100 $\mathrm{mV}$ after the potential was scanned to $0 \mathrm{mV}$, where the bulk deposition of Te occurred. Since all the desorption current peaks appeared at potentials more positive than $+100 \mathrm{mV}$, the deposited bulk and upd layers of Te still existed on the Au(111) surface. The original 3-fold pattern in Figure 3a vanished and only the other 3-fold pattern which appeared in Figure $3 b$ was observed with a much smaller SH intensity. After the potential was scanned to $800 \mathrm{mV}$, where all the deposited layers of Te were desorbed, the $\mathrm{SH}$ rotational pattern became the same

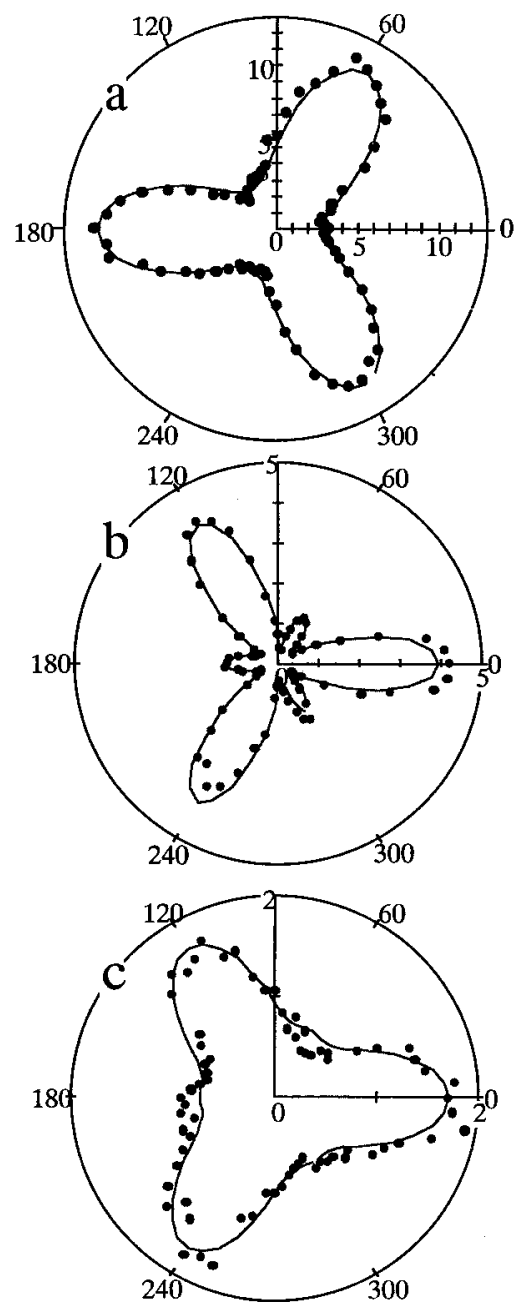

Figure 3. SH rotational patterns of the $\mathrm{Au}(111)$ electrode surface in $0.1 \mathrm{M} \mathrm{HClO}_{4}+0.5 \mathrm{mM} \mathrm{TeO}_{2}$ at (a) $+800 \mathrm{mV}$, (b) $+150 \mathrm{mV}$, and (c) $+100 \mathrm{mV}$. Polarization: p-in/p-out. Circles show data points, and solid line indicates the theoretical fit.

TABLE 2: Fitting Parameters for p-in/p-out SH Rotational Anisotropies of the $\mathrm{Au}(111)$ Electrode

\begin{tabular}{lcl}
\hline \multicolumn{1}{c}{ solution } & $\begin{array}{c}\text { electrode potential } \\
(\mathrm{mV})\end{array}$ & $\mathbf{d}_{\mathrm{pp}}{ }^{(3)} / \mathbf{a}_{\mathrm{pp}}{ }^{(\infty)}$ \\
\hline $0.1 \mathrm{M} \mathrm{HClO}_{4}$ & 800 & $0.58 \mathrm{e}^{\mathrm{i} 47^{\circ}}$ \\
& 150 & $0.70 \mathrm{e}^{\mathrm{i} 40^{\circ}}$ \\
$0.1 \mathrm{M} \mathrm{HClO}_{4}+0.5 \mathrm{mM} \mathrm{TeO}_{2}$ & 800 & $0.60 \mathrm{e}^{\mathrm{i} 49^{\circ}}$ \\
& 150 & $1.78 \mathrm{e}^{\mathrm{i} 125^{\circ}}$ \\
& 100 & $0.54 \mathrm{e}^{\mathrm{i} 118^{\circ}}$
\end{tabular}

as that shown in Figure 3(a), indicating that the bare $\mathrm{Au}(111)$ surface was recovered and the electrochemical deposition/ desorption of Te was a reversible process. The parameters $\mathbf{a}^{(\infty)}$, $\mathbf{d}^{(3)}$, and the phase difference were obtained by fitting the $\mathrm{SH}$ rotational data presented in Figures 2 and 3 to the theoretical eq $1 \mathrm{a}$ for the $\mathrm{p}$-in/p-out $\mathrm{SH}$ rotational patterns and are summarized in Table 2.

Figure 4 shows the $\mathrm{SH}$ rotational patterns obtained under other polarization conditions, i.e., s-in/p-out and s-in/s-out polarization combinations, before and after the formation of the first upd layer of Te on the $\mathrm{Au}(111)$ surface. For the s-in/p-out and s-in/ s-out polarization combinations, patterns referring to the 3-fold symmetry were obtained. These patterns indicate the $C_{3 v}$ symmetry of the surface. While the shape for the $s$-in/p-out $\mathrm{SH}$ rotational pattern changed as a result of the upd of Te, the shape of the s-in/s-out SH rotational pattern did not change with 

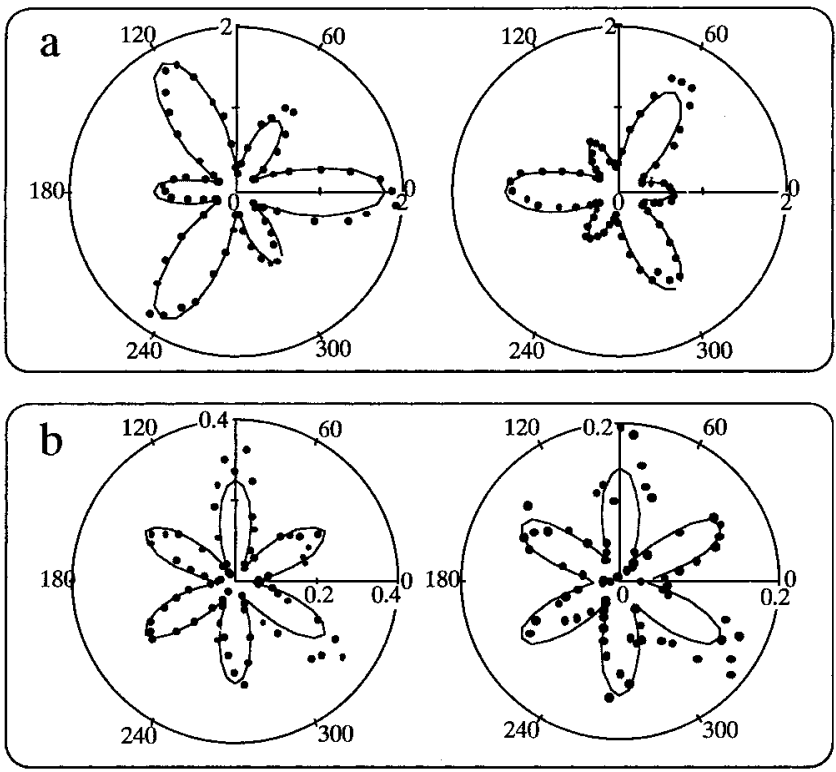

Figure 4. SH rotational patterns of the $\mathrm{Au}(111)$ electrode surface in $0.1 \mathrm{M} \mathrm{HClO}_{4}+0.5 \mathrm{mM} \mathrm{TeO}_{2}$ before (left) and after (right) the first upd of Te for (a) s-in/p-out and (b) s-in/s-out polarization combinations. Circles show data points, and solid line indicates the theoretical fit.

TABLE 3: Fitting Parameters for s-in/p-out and s-in/s-out SH Rotational Anisotropies of the $\mathrm{Au}(111)$ Electrode in 0.1 $\mathrm{M} \mathrm{HClO}_{4}+0.5 \mathrm{mM} \mathrm{TeO}_{2}$ Solution

\begin{tabular}{lcl}
\hline polarization & $\begin{array}{c}\text { electrode potential } \\
(\mathrm{mV})\end{array}$ & \multicolumn{1}{c}{ fitting parameters } \\
\hline s-in/p-out & 800 & $\mathbf{d}_{\mathrm{sp}}{ }^{(3)} / \mathbf{a}_{\mathrm{sp}}{ }^{(\infty)}=-1.9 \mathrm{e}^{\mathrm{i} 70^{\circ}}$ \\
& 150 & $\mathbf{d}_{\mathrm{sp}}{ }^{(3)} / \mathbf{a}_{\mathrm{sp}}{ }^{(\infty)}=-1.4 \mathrm{e}^{1114^{\circ}}$ \\
s-in/s-out & 800 & $\mathbf{d}_{\mathrm{ss}}{ }^{(3)}=0.43$ \\
& 150 & $\mathbf{d}_{\mathrm{ss}}{ }^{(3)}=0.32$
\end{tabular}

the upd of Te. The fitting parameters for these patterns obtained using eqs $1 \mathrm{~b}$ and $1 \mathrm{~d}$ are summarized in Table 3 .

\section{Discussion}

5.1. First Te upd. The $\mathrm{p}$-in/p-out SH rotational anisotropy at $+150 \mathrm{mV}$ in a solution containing $0.1 \mathrm{M} \mathrm{HClO}_{4}$ and 0.5 $\mathrm{mM} \mathrm{TeO}{ }_{2}$ was significantly different from that in pure $0.1 \mathrm{M}$ $\mathrm{HClO}_{4}$ solution at the same potential. The change of the $\mathrm{SH}$ rotational anisotropy should be due to the first upd of Te and is clearly reflected in the change of the parameters $\mathbf{d}_{\mathrm{pp}}{ }^{(3)}$ and $\mathbf{a}_{\mathrm{pp}}{ }^{(\infty)}$. The magnitude of $\mathbf{d}_{\mathrm{pp}}{ }^{(3)} / \mathbf{a}_{\mathrm{pp}}{ }^{(\infty)}$ changed from $\left|\mathbf{d}_{\mathrm{pp}}{ }^{(3)} / \mathbf{a}_{\mathrm{pp}}{ }^{(\infty)}\right|<1$ to $\left|\mathbf{d}_{\mathrm{pp}}{ }^{(3)} / \mathbf{a}_{\mathrm{pp}}{ }^{(\infty)}\right|>1$, and the phase difference between $\mathbf{a}_{\mathrm{pp}}{ }^{(\infty)}$ and $\mathbf{d}_{\mathrm{pp}}{ }^{(3)}$ changed from $47^{\circ}$ at the bare gold electrode to $125^{\circ}$ after the first upd of Te. In addition, the SH intensity became much weaker than that from the bare $\mathrm{Au}(111)$. These changes were caused by a change in geometric and electronic structures of the $\mathrm{Au}(111)$ surface by the first upd of Te.

Previous studies dealing with the 1064-nm-excited SH response during electrochemical deposition on $\mathrm{Au}(111)$ surfaces ${ }^{41-43}$ showed that the deposition of metals such as Ag and $\mathrm{Cu}$ with electronic structure similar to Au was accompanied by a very small change in the shape of the SH rotational pattern, i.e., the small change of $\left|\mathbf{d}_{\mathrm{pp}}{ }^{(3)} / \mathbf{a}_{\mathrm{pp}}{ }^{(\infty)}\right|$ and the small phase difference change. On the other hand, deposition of $\mathrm{Pb}$ or $\mathrm{Tl}$ at a coverage of $\theta=1$ caused a much greater change in the magnitude of $\mathbf{d}_{\mathrm{pp}}{ }^{(3)} / \mathbf{a}_{\mathrm{pp}}{ }^{(\infty)}$ as a result of the decrease in the anisotropic contribution, $\mathbf{d}_{\mathrm{pp}}{ }^{(3)}$. The change of the phase difference was also small in this case.

The change of the SH rotational anisotropy during the first upd of $\mathrm{Te}$ on $\mathrm{Au}(111)$ was different from those for both the $\mathrm{Ag}$ or $\mathrm{Cu} / \mathrm{Au}(111)$ and $\mathrm{Pb}$ or $\mathrm{Tl} / \mathrm{Au}(111)$ systems as both $\mid \mathbf{d}_{\mathrm{pp}}{ }^{(3)} /$ $\mathbf{a}_{\mathrm{pp}}{ }^{(\infty)} \mid$ and the phase difference significantly changed. To explain this result, two different models can be considered. In the first model, the strong SHG response generated at the Au(111) surface was quenched as a result of the upd of Te. In the second model, the SHG response was considered to be generated not only from the $\mathrm{Au}(111)$ surface but also from the adsorbed Te layer.

5.1.1. Quenching Model. Information about the isotropic term, $\mathbf{a}_{\mathrm{pp}}{ }^{(\infty)}$, can be obtained from the p-in/p-out SH intensity at the azimuthal angle of $30^{\circ}$ as only $\mathbf{a}_{\mathrm{pp}}{ }^{(\infty)}$ contributes to the $\mathrm{SH}$ response under these conditions. The decrease in the $\mathrm{p}$-in/ p-out SH intensity at the azimuthal angle of $30^{\circ}$, i.e., $\mathbf{a}_{\mathrm{pp}}^{(\infty)}$, with the first Te upd as shown in Figure 3a,b supports the hypothesis proposed in the previous report, ${ }^{27}$ in which the decrease in the $\mathrm{SH}$ intensity during the first $\mathrm{Te}$ upd on a polycrystalline $\mathrm{Au}$ electrode was examined. Thus, the large nonlinear susceptibility of the Au surface at the SH photon energy of $2.33 \mathrm{eV}$ as a result of the coupling of the $\mathrm{SH}$ photon with the $5 \mathrm{~d}-6 \mathrm{~s}$ interband transition $(2.25 \mathrm{eV})^{42,41,44}$ was quenched by the formation of the first upd layer of Te. In other words, the resonant condition at this energy was lost by the first upd of Te.

On the other hand, anisotropic response, $\mathbf{d}^{(3)}$, did not significantly change. The decrease of $\mathbf{d}_{\mathrm{ss}}{ }^{(3)}$ with the first upd of Te was about 20\%, as shown in Table 3. Since anisotropic responses in $\mathrm{p}$-in/p-out and s-in/s-out polarization combinations are proportional to $\chi^{(2)}{ }_{x x x}, \mathbf{d}_{\mathrm{pp}}(3)$ can be considered to decrease by the same ratio during the first Te upd. Thus, the first Te upd causes a weak decrease in the $\mathbf{d}^{(3)}$ term and the resonantto-nonresonant transition should occur in the $\mathbf{a}^{(\infty)}$ term.

Such a resonant-to-nonresonant transition was more evident for the change in the phase difference between $\mathbf{a}_{\mathrm{pp}}{ }^{(\infty)}$ and $\mathbf{d}_{\mathrm{pp}}{ }^{\left({ }^{(3)}\right.}$. The change in the phase difference from $+800 \mathrm{mV}$ to +150 $\mathrm{mV}$ in $\mathrm{HClO}_{4}$ solution was merely $10^{\circ}$, although the desorption of perchlorate anions and surface reconstruction of $\mathrm{Au}(111)$ occur within this potential region. This result is in agreement with the conclusion by Pettinger and his co-workers that the phenomena such as the adsorption/desorption of anions and surface reconstruction do not significantly alter surface electronic states. ${ }^{41}$ They proposed that the observed small change in the phase difference was due to the change in the surface charge density. On the other hand, the change in the phase difference from $+800 \mathrm{mV}$ to $+150 \mathrm{mV}$ in the electrolyte solution containing Te observed in this study was more than $60^{\circ}$. The drastic change in the relative phase difference with the first upd of Te strongly suggests the resonant-to-nonresonant transition in the $\mathbf{a}^{(\infty)}$ term.

If the resonant-to-nonresonant transition only in the $\mathbf{a}^{(\infty)}$ term caused the change of the rotational anisotropy pattern after the first Te upd, a contradiction arises. Since the above-mentioned resonance between the SH photon and the $5 \mathrm{~d}-6 \mathrm{~s}$ interband transition can be allowed in the in-plane direction, the resonantto-nonresonant transition should be clear in the $\mathbf{d}_{\mathrm{pp}}{ }^{(3)}$ term. The observation of the resonant-to-nonresonant transition only in the $\mathbf{a}^{(\infty)}$ term means that the additional resonance which lies in the out-of-plane direction exists at the bare $\mathrm{Au}(111)$ surface. The electron transition from the intrinsic surface states around $\Gamma$ to the bulk empty states of $\mathrm{Au}(111)$ surface can be accessible for the resonance since such a transition can be induced only by photons polarized in the out-of-plane direction. ${ }^{41}$ To confirm the contribution of this resonance, however, the spectroscopic studies should be carried out. 
5.1.2. Model Including the Contribution of Adsorbate to SH Rotational Anisotropy. In the above description, the changes in the SH anisotropy were treated as a result of the modification of the surface electronic features of $\mathrm{Au}(111)$. In other words, the origin of the SHG signal was assumed to be the electronic response only at the gold surface even after the first Te upd. If the contribution of the adsorbate or the adsorbate-substrate bond can dominate in the SHG response, a different approach to explain the change in $\mathrm{SH}$ rotational anisotropy upon $\mathrm{Te}$ deposition should be taken. Kim et al. employed an analyzing method of $\mathrm{SH}$ rotational anisotropy to determine the adsorbate structure of 2,5-dihydroxythiophenol (DHTP) molecules on Au(111). ${ }^{45}$ They considered a surface phase formed by adsorption attenuates the surface contribution of the substrate itself but also generates an additional contribution to the $\mathrm{SH}$ rotational anisotropy. The nonlinear susceptibility of the adsorbatemodified surface, $\chi_{\mathrm{s}}^{\prime}(2)$, was expressed as

$$
\chi_{\mathrm{S}}^{(2)}=\alpha \chi_{\mathrm{S}}^{(2)}+\chi_{\mathrm{A}}^{(2)}
$$

where $\alpha$ is a substrate attenuation factor and $\chi_{\mathrm{s}}{ }^{(2)}$ and $\chi_{\mathrm{A}}{ }^{(2)}$ are the nonlinear susceptibilities of the bare surface and the adsorbate, respectively. They chose the excitation frequency of $560 \mathrm{~nm}$ so that the resonance of $2 \omega$ frequency with the intramolecular electronic transition of adsorbed DHTP occurred and the molecular contribution became comparable to the substrate contribution.

Equation 3 can be applied without further modification for the surface nonlinear susceptibility of the Te-modified $\mathrm{Au}$ surface. Since $I(2 \omega) \propto\left(\chi^{\prime} \mathrm{s}^{(2)}\right)^{2}$ and the experimental results clearly show that the SHG signal ascribed to the adsorbate is 3 -fold, $I(2 \omega)$ can be written as ${ }^{45}$

$$
\begin{aligned}
& I(2 \omega) \propto\left|\alpha \chi_{\mathrm{S}}{ }^{(2)}+\chi_{\mathrm{A}}^{(2)}\right|^{2}= \\
& \alpha^{2}\left|\chi_{\mathrm{S}}^{(2)}\right|^{2}+\left|\chi_{\mathrm{A}}{ }^{(2)}\right|^{2}+2 \alpha\left|\chi_{\mathrm{S}}{ }^{(2)}\right|\left|\chi_{\mathrm{A}}{ }^{(2)}\right| \cos \Delta
\end{aligned}
$$

where the equations for the rotational dependence of the nonlinear susceptibility of both the substrate and the adsorbate are given by eqs 1 for each input and output polarization condition. For example, under the $\mathrm{p}$-in/p-out polarization condition,

$$
\begin{aligned}
& \chi_{\mathrm{s}}{ }^{(2)}=\mathbf{a}_{\mathrm{pp}}{ }^{(\infty)}+d_{\mathrm{pp}}{ }^{(3)} \cos (3 \phi), \chi_{\mathrm{A}}{ }^{(2)}= \\
& \mathbf{a}_{\mathrm{pp}}^{\prime}{ }^{(\infty)}+\mathbf{d}_{\mathrm{pp}}{ }^{(3)} \cos \{3(\phi-\beta)\}
\end{aligned}
$$

and under the s-in/s-out condition,

$$
\chi_{\mathrm{s}}{ }^{(2)}=\mathbf{d}_{\mathrm{ss}}{ }^{(3)} \sin (3 \phi), \quad \chi_{\mathrm{A}}{ }^{(2)}=\mathbf{d}_{\mathrm{ss}}{ }^{(3)} \sin \{3(\phi-\beta)\}
$$

where $\beta$ is the difference in the azimuthal angle $\phi$ between the surface structure of the substrate and that of the adsorbate layer. As stated above, the coefficients $\mathbf{a}^{(\infty)}, \mathbf{d}^{(3)}, \mathbf{a}^{\prime(\infty)}$, and $\mathbf{d}^{\prime(3)}$ are all complex numbers. Hence, three relative phase differences, $\Theta$ between $\mathbf{a}^{(\infty)}$ and $\mathbf{d}^{(3)}, \Theta^{\prime}$ between $\mathbf{a}^{\prime(\infty)}$ and $\mathbf{d}^{\prime(3)}$, and $\Delta$ between $\mathbf{a}^{(\infty)}$ and $\mathbf{a}^{\prime(\infty)}$, must be evaluated.

The SH rotational anisotropy of the $\mathrm{Au}(111)$ surface biased at $+150 \mathrm{mV}$ with the first upd layer of Te was fitted to eq 4 to obtain various parameters. The values of $\mathbf{a}^{(\infty)}$ and $\mathbf{d}^{(3)}$ of clean $\mathrm{Au}(111)$ biased at $+150 \mathrm{mV}$ in pure electrolyte were substituted into eq 5 to avoid the electric field induced enhancement of SHG. As shown in Table 2, a fitting of the data yielded for bare $\mathrm{Au}(111) \mathbf{a}_{\mathrm{pp}}\left({ }^{\infty}\right) / \mathbf{d}_{\mathrm{pp}}{ }^{(3)}=1.43 \mathrm{e}^{\mathrm{i} 57^{\circ}}$ under the $\mathrm{p}$-in/p-out polarization condition. Substitution of these values and fitting of the data for the first upd Te layer on $\mathrm{Au}(111)$ gave $\mathbf{a}_{\mathrm{pp}}{ }^{\left({ }^{\infty}\right) /}$ $\mathbf{d}_{\mathrm{pp}}^{\prime}{ }^{(3)}=0.49 \mathrm{e}^{153^{\circ}}, \alpha=-0.65, \beta=58.8^{\circ}$, and $\Delta=-36.4^{\circ}$.

The SH rotational anisotropy under the s-in/p-out polarization condition was analyzed in a similar manner. The statistical fitting to the s-in/p-out $\mathrm{SH}$ rotational anisotropy for the first upd Te layer on $\mathrm{Au}(111)$ yielded $\mathbf{a}_{\mathrm{sp}}^{\prime(\infty)} / \mathbf{d}_{\mathrm{sp}}{ }^{(3)}=-0.914 \mathrm{e}^{\mathrm{i} 55^{\circ}}$, $\alpha=-0.52, \beta=58.7^{\circ}$, and $\Delta=-140^{\circ}$. The most important parameter is $\beta$, which provides information about surface geometry. The values of $\alpha$, which are smaller than unity under both polarization conditions, confirm the decrease in the contribution of the $\mathrm{Au}(111)$ surface. This decrease in the isotropic $\mathrm{SH}$ response should be attributed to simple electron scattering by the Te adsorbate at the surface in the present model. Other parameters such as $\Delta$ contain the Fresnel factors and the relative phase and are difficult to discuss.

The adsorbate structure of Te on Au single-crystalline surfaces has been already investigated by ex situ LEED and STM and in situ STM and AFM. ${ }^{15,16,21,22}$ The reported structure of the first upd layer of $\mathrm{Te}$ on $\mathrm{Au}(111)$ is the $(\sqrt{ } 3 \times \sqrt{ } 3) R 30^{\circ}$ surface structure. The value $\beta \cong 60^{\circ}$, obtained by the theoretical fitting of the SH rotational anisotropy for $\mathrm{Au}(111)$ covered with the first upd layer of Te does not coincide with the angle of rotation between the substrate $\mathrm{Au}$ lattice and the upd Te expected for the $(\sqrt{ } 3 \times \sqrt{ } 3) R 30^{\circ}$-Te structure. These results indicate that the contribution of an additional surface phase did not affect the surface nonlinear polarization.

It is clear at this stage that the change in the $\mathrm{SH}$ rotational anisotropy by the first upd of Te was derived only due to the change in the surface electronic structure of $\mathrm{Au}(111)$ and not due to the electronic contributions of both $\mathrm{Au}(111)$ and the Te layer.

5.2. Bulk Te Deposition. After the bulk deposition of Te, a further decrease in the SH intensity was observed. The magnitude of $\mathbf{d}_{\mathrm{pp}}{ }^{(3)} / \mathbf{a}_{\mathrm{pp}}{ }^{(\infty)}$ changed from $\left|\mathbf{d}_{\mathrm{pp}}{ }^{(3)} / \mathbf{a}_{\mathrm{pp}}{ }^{(\infty)}\right|>1$ to $\left|\mathbf{d}_{\mathrm{pp}}{ }^{(3)} / \mathbf{a}_{\mathrm{pp}}{ }^{(\infty)}\right|<1$ again, but the phase difference was changed only 58 from that of $\mathrm{Au}(111)$ covered with the first upd layer of Te. Thus, the change corresponding to the bulk deposition seemed to be solely due to the decrease in the anisotropic contribution, $\mathbf{d}_{\mathrm{pp}}{ }^{(3)}$.

In the previous studies mentioned above,${ }^{41-43}$ deposition of $\mathrm{Pb}$ or $\mathrm{Tl}$ at a coverage of $\theta=1$ causes a much greater change in the magnitude of $\mathbf{d}_{\mathrm{pp}}{ }^{(3)} / \mathbf{a}_{\mathrm{pp}}{ }^{(\infty)}$ because of the decrease in the anisotropic contribution, $\mathbf{d}_{\mathrm{pp}}{ }^{(3)}$. The change in the $\mathrm{SH}$ rotational anisotropy of the $\mathrm{Pb}$ or $\mathrm{Tl} / \mathrm{Au}(111)$ system was explained by the existence of two different domain structures of the overlayer, which are rotated by angles of $+\Phi$ and $-\Phi$ from the gold lattice. ${ }^{41}$ The authors concluded that because the same amount of these domains existed, the surface symmetry became nearly isotropic and the anisotropic response, $\mathbf{d}_{\mathrm{pp}}{ }^{(3)}$, became small. In the present case of $\mathrm{Te} / \mathrm{Au}(111)$, the change in the $\mathrm{SH}$ rotational anisotropy during the bulk deposition on the $\mathrm{Au}(111)$ surface with the first upd layer resembled that for the case of $\mathrm{Pb}$ or $\mathrm{Tl}$ deposition on $\mathrm{Au}(111)$. Another possible cause for the decrease in $\mathbf{d}_{\mathrm{pp}}{ }^{(3)}$ is the three -dimensional growth of Te, which results in a more isotropic surface morphology, as was proposed for the bulk Te layers that were formed via the Stranski-Krastanov mechanism, ${ }^{22}$ i.e., three-dimensional growth after the two dimensional formation of the first monolayer.

\section{Conclusion}

Electrochemical deposition of $\mathrm{Te}$ on an $\mathrm{Au}(111)$ electrode surface was investigated using SHG measurements. The $\mathrm{SH}$ rotational anisotropy significantly changed with the first upd of Te. Bulk Te deposition attenuated the anisotropic character 
of the overall surface symmetry, as expected from the previous SHG studies on $\mathrm{Tl} / \mathrm{Au}(111)$ and $\mathrm{Pb} / \mathrm{Au}(111)$.

The change in the $\mathrm{SH}$ rotational anisotropy during the first upd of Te was examined using two different models. The first and second models considered the contribution of only the $\mathrm{Au}-$ (111) surface and both the $\mathrm{Au}(111)$ and the adsorbed Te layer to the SHG response, respectively. The analysis for the latter model resulted in a rotation angle of $60^{\circ}$ for the deposited Te layer against the $\mathrm{Au}(111)$ lattice. This value was inconsistent with the expected adsorbate structure of Te, i.e., $(\sqrt{ } 3 \times \sqrt{ } 3)$ $R 30^{\circ}$. Thus, the latter model seemed not to be suitable for explaining the present results. To clarify the resonant-tononresonant transition at the $\mathrm{Au}(111)$ surface during $\mathrm{Te}$ upd, which is proposed in the former model, more quantitavely, in situ monitoring of the real phase shift ${ }^{42,46}$ of each tensor element during the first upd of Te is required.

Acknowledgment. This work was partially supported by a Grant-in-Aid for Priority Area Research on "Electrochemistry of Ordered Interfaces" (No. 09237101) from the Ministry of Education, Science, Sports and Culture, Japan. I.Y. acknowledges the Japan Society for the Promotion of Science for the JSPS Research Fellowship for Young Scientists.

\section{References and Notes}

(1) Kolb, D. M. In Advances in Electrochemistry and Electrochemical Engineering Gerischer, H., Tobias, H., Eds.; Wiley: New York, 1978; Vol. 11 .

(2) (a) Kolb, D. M.; Przasnyski, M.; Gerischer, H. J. Electroanal. Chem. 1974, 54, 25. (b) Kolb, D. M.; Przasnyski, M.; Gerischer, H. Surf. Sci. 1974, 43, 662

(3) Leiva, E. Electrochim. Acta 1996, 41, 2185.

(4) Adzic, R. In Advances in Electrochemistry and Electrochemical Engineering; Gerischer, H., Tobias, H., Eds.; Wiley: New York, 1984; Vol. 13 .

(5) Magnussen, O. M.; Hotlos, J.; Nichols, R. J.; Kolb, D. M.; Behm, R. J. Phys. Rev. Lett. 1990, 64, 2929.

(6) Magnussen, O. M.; Hotlos, J.; Beitel, G.; Kolb, D. M.; Behm, R. J. J. Vac. Sci. Technol. B 1991, 9, 969.

(7) Hachiya, T.; Honbo, H.; Itaya, K. J. Electroanal. Chem. 1991, 315 , 275.

(8) Hamelin, A.; Lipkowski, J. J. Electroanal. Chem. 1984, 171, 317.

(9) Pauling, H. J.; Juettner, K. Electrochim. Acta 1992, 37, 2237.

(10) Manne, S.; Hansma, P. K.; Massie, J.; Elings, V. B. Gewirth, A. A. Science 1991, 251, 183 .

(11) Gregory, B. W.; Norton, M. L.; Stickney, J. L. J. Electroanal. Chem. 1990, $293,85$.

(12) Gregory, B. W.; Stickney, J. L. J. Electroanal. Chem. 1991, 300, 543.

(13) Gregory, B. W.; Suggs, D. W.; Stickney, J. L. J. Electrochem. Soc. 1991, 138, 1279 .

(14) Suggs, D. W.; Stickney, J. L J. Phys. Chem. 1991, 92, 10056.

(15) (a) Suggs, D. W.; Stickney, J. L Surf. Sci. 1993, 290, 362. (b) Suggs,

D. W.; Stickney, J. L Surf. Sci. 1993, 290, 375.
(16) (a) Goetting, L. B.; Huang, B .M.; Lister, T. E.; Stickney, J. L. Electrochim. Acta 1995, 40, 143. (b) Huang, B. M.; Colletti, L. P.; Gregory, B. W.; Stickney, J. L. J. Electrochem. Soc. 1995, 142, 3007.

(17) Colletti, L. P.; Teklay, D.; Stickney, J. L. J. Electroanal. Chem. 1994, 369, 145.

(18) (a) Villegas, I.; Stickney, J. L. J. Electrochem. Soc. 1992, 139 686. (b) Villegas, I.; Stickney, J. L. J. Vac. Sci. Technol. A 1992, 10, 3032.

(19) Lister, T. E.; Huang, B. M.; Herrick II, R. D.; Stickney, J. L. J. Vac. Sci. Technol. B 1995, 13, 1268.

(20) (a) Demir, U.; Shannon, C. Langmuir 1994, 10, 2794. (b) Demir, U.; Shannon, C. Langmuir 1995, 12, 594.

(21) Wan, L. J.; Sashikata, K.; Yau, X. L.; Itaya, K. Abstracts of The Meeting of the Japanese Electrochemical Society, Yokohama, September 19-20, 1994

(22) Ikemiya, N.; Iwai, D.;. Yamada, K; Vidu, R.; Hara, S. Surf. Sci. 1996, 369, 199.

(23) Shen, Y. R. In The Principles of Nonlinear Optics; Wiley: New York, 1984.

(24) Richmond, G. L.; Robinson, J. M.; Shannon, V. L. Prog. Surf. Sci. 1988, 28,1 .

(25) Corn, R. M.; Higgins, D. A. Chem. Rev. 1994, 94, 107.

(26) Heinz, T. F. In Nonlinear Surface Electromagnetic Phenomena,; Ponath, H.-E., Stegeman, G. I., Eds.; Elsevier: Amsterdam, 1991.

(27) Yagi, I.; Lantz, J. M.; Nakabayashi, S.; Corn, R. M.; Uosaki, K. J. Electroanal. Chem. 1996, 401, 95.

(28) (a) Mizrahi, V.; Sipe, J. E. J. Opt. Soc. Am. B 1988, 5, 660. (b)

Sipe, J. E.; Moss, D. J.; van Driel, H. M. Phys. Rev. B 1987, 35, 1129.

(29) Guyot-Sionnest, P.: Chen, W.; Shen, Y. R. Phys. Rev. B 1986, 33,8254 .

(30) Fischer, P. R.; Daschbach, J. L.; Gragson, D. E.; Richmond, G. L. J. Vac. Sci. Technol. A 1994, 12, 2617.

(31) Mirwald, S.; Pettinger, B.; Lipkowski, J. Surf. Sci. 1995, 335, 264.

(32) Butcher, P. N.; Cotter, D. In The Elements of Nonlinear Optics; Cambridge University Press: Cambridge, 1990.

(33) Clavilier, J.; Faure, R.; Guinet, G.; Durand, R. J. Electroanal. Chem. 1980, 107, 205.

(34) Motoo, S.; Furuya, N. Ber. Bunsen-Ges. Phys. Chem. 1987, 91, 457.

(35) Mori, E.; Baker, C. K.; Reynolds, J. R.; Rajeshwar, K. J. Electroanal. Chem. 1988, 252, 441.

(36) Traore, M.; Modolo, R.; Vittori, O. Electrochim. Acta 1988, 33, 991.

(37) Rosamilia, J. M.; Miller, B. J. Electroanal. Chem. 1986, 215, 261.

(38) Friedrich, A.; Pettinger, B.; Kolb, D. M.; Lüpke, G.; Steinhoff, R.; Marowski, G. Chem. Phys. Lett. 1989, 163, 123.

(39) Lüpke, G.; Marowski, G.; Steinhoff, R.; Friedrich, A.; Pettinger, B.; Kolb, D.M. Phys. Rev. B 1990, 41, 6913.

(40) Pettinger, B.; Lipkowski, J.; Mirwald, S.; Friedrich, A. J. Electroanal. Chem. 1992, 329, 289.

(41) Koos, D. A.; Richmond, G. L. J. Phys. Chem. 1992, 96, 3770

(42) Koos, D. A.; Richmond, G. L J. Phys. Chem. 1990, 93, 869.

(43) Koos, D. A. J. Electroanal. Chem. 1989, 136, 218C.

(44) Johnson, P. B.; Christy, R.W. Phys. Rev. B 1972, 6, 4370.

(45) Kim, S.; Zhao, M.; Scherson, D. A.; Choi, K. J.; Bae, I. T. J. Phys. Chem. 1994, 98, 9383.

(46) (a) Chang, R. K.; Ducuing, J.; Bloembergen, N. Phys. Rev. Lett. 1965, 15, 6. (b) Schwarzberg, E.; Berkovic, G.; Marowsky, G. Appl. Phys. A 1994, 59, 631 . 